\title{
Study and Application of 220kV Substation Relay Protection Training System
}

\author{
Yuan Ting, Zhang He, Liu Shusen \\ Panjin Power Supply Company, Liaoning Electric Power Company Limited, State Grid, China, \\ 16295036@qq.com
}

Keywords: substation intelligent training; digital-analog hybrid simulation; embedded fault simulation;

\begin{abstract}
With the large-scale application of power electronics technology and computer technology in grid operation, automation level of the grid production and operation maintenance in China is increasingly higher, which also proposes higher requirements on the professional theory and skills of frontline staff in grid operation. Relay protection and automation devices are an important part for the safe and stable operation of power grid and also key tools and methods to protect the safety of electrical equipment. Therefore, how to quickly improve the technological level of front-line staff in grid operation and maintenance so that they can meet the needs of automotive and informative development of power grid has become a key obstacle for the safe and stable operation of power grid.At present, theoretical and technical trainingsfor front-line staff of power system operation and maintenance are mainly carried out in the form of regular experts and electricity universities training courses organized by the enterprises, and teaching by certain elder staff at the scene. However, there are certain limitations in the above two training methods in that they cannot meet at the same time the theoretical and practical and technical development needs and that the training results are not always satisfying. Therefore, in order to adapt to the rapid development of power system in China, it is urgent to establish a sound and systematic power system relay protection and automation operation and maintenance personnel training system, which should include basic theory training, practice operation and other cutting-edge power technology overview, so as to make it possible for the frontline staff to learn theoretical knowledge and at the same time have a grasp of the power system development trend and new technologies probably to come. It should ensure that the frontline staff understand and learn new methods for relay protection and automatic detection.
\end{abstract}

\section{Analyzing the Present Status of Relay Protection System}

Professional training for relay protection has been in the mode of single protective device adjustment since 2000 , testing the reliability, timeliness and sensitivity of the protection device by adding simulation value on the protective tester, or setting certain artificial defects and anomaliesin the second line on the screen if the training is of greater difficulty. However, this training mode can hardly cover comprehensive experimental programs like complex faults, protection cooperation and secondary circuit fault analysis, which makes the training of greatly unsatisfying and less targeted. Therefore, it is necessary to expand the training content and improve the training quality. With the continuous development of simulation technology, grid simulation technology has been increasingly applied in training as it can make the training more close to the actual scene, provide the trainees a more realistic training environment and get recognition from trainers and trainees.

Training system for relay protection is in fact a simulation model of the actual operation situation of power grid, practicing practical operation on the simulation model to study on the changes of amount of electric power in the power system and judgment and countermeasures against faults.

Simulation studies of power system in foreign countries began as early as the 1970s, when the United States developed the first thermal power simulation system in the world. Later, the UK and Japan also launched its own first set of power simulation system. In the 1980s, Tsinghua University 
successfully developed the first set of training system for thermal power generating units in China. In the late 1980s, Dalian, Guangzhou, Hebei and other training centers have successfully developed their own substation simulation training systems, which includerelay protection training system. Domestic colleges and universities also carried out numerousresearch projects on simulation system. This research program is also participated by a senior professor fromNCEPU

China Electric Power Research Institute classifies the development history of power system simulation into the following stages:

Stage 1 is a smaller version of the power system, whichresizes the actual operating devices into a smaller one.

Stage 2 is a real-time simulation system of the power system by simulating the electronic devices with electronic technologies.

Stage 3 is a hybrid simulation system combining the expanded working environment of real devices by computer technologies and training functions as complementary.

Stage 4 is the development of a real-time digital simulation of the power system by using the pure digital simulation training system realized by computer technologies.

Stage 5 is a real-time hybrid simulation system combining the expanded working environment of real devices by computer technologies and training functions as complementary.

Thesimulation training systemplays a significant roleintrainings for electrical workers. Substation simulation training system has been applied in the grid system in China for many years. The simulation system has been gradually improved and the simulation effect is becoming increasingly realistic so that some simulation design has been adopted and put into practical production by the power system.

\section{Classification and Characteristics of Relay Protection Training System}

Researches on relay protection training simulation system have a long history and can be classified according to the following standards.

According to whether there isa hardware panel

According to whether there is a hardware panel as the output support of the software, simulation system of substations can be classified as "hardware" simulation, "software" simulation and "hybrid" simulation.

Hardware simulation refers to simulate the primary device room and secondary protection circuit chamber with a real hardware panel, showing the calculated simulation information output by the software on the hardware part. Similarly, simulation training system uses real devices to make the simulation operation direct and realistic. However, real devices are not economic and electrical devices are generally expensive, which means that the investment in simulation training system is higher and the operation and that maintenance of the real devices would consume certain manpower and material resources. Restriction of real devices makes it quite difficult to further develop hardware simulation.

Software simulation uses microcomputer processors to simulate the logic of relay protection, switching operation sequence and changing situation of electrical power in the power system. Primary and secondary equipment of the substation are all simulated by microcomputer processors and there is no real device operating in the whole system. It is advantageousinits low investment and convenient maintenance and the function to expand automatically on the microprocessor. It is disadvantageous in its power authenticity and failure in simulating the impact of on-site weather and other natural factors on the system.

Hybrid simulation combines the software simulation and hardware simulation, simulating equipment of great investment and inconvenient to expand with microprocessor, such as transformer, mutual inductor, capacity and other primary equipment, and supplement equipment of little investment and convenient to maintain with real devices. Hybrid simulation combines the advantages of the above two, improving authenticity of the training process and also reducing investment in training system and maintenance of the equipment. 


\section{According to the scale of simulation}

Simulation of a single substation: only simulate a substation and make software model of the primary parts. This type of simulation is small in scale and of low cost. However, it ignores the external grid and fails to take the impact of the whole power system on operation of the substation into consideration; it has certain limitations and of little training value for modern grid staff.

Simulation of several substations: simulate several substations and connect them into a system. This type of simulation should take many factors into consideration, such as: cooperating situation of the substations, operating situation of each substation and the real-time working situation of each substation in issue cooperation. However, this type of simulation takes the highest cost and the longest development cycle.

\section{Content of Relay Protection Training System in Substations}

After the $220 \mathrm{kV}$ substation relay protection training system is adopted, the main work is to teaching the relay protection staff, substation operating staff and direct current equipment maintenance staff technical skills. Therefore, the change of function makes it necessary to have special design. Its main function should include the following aspects of training content:

(1) Switching operation training of disconnector and circuit breaker;

(2) Training on the filling-in of operating ticket and job ticket;

(3) Routine equipment maintenance and inspection training;

(4) Training on abnormalityanalysis and processing methods and ways;

(5) Secondary circuit operation training;

(6) Secondary monitoring and recording training;

(7) Automatic judgment of misoperations and corrective measures;

(8) Student examination system;

Relay protection training system of the substation simulates model and power flow of the primary equipment in the virtual substation, and connects the secondary equipment in the real substation, including protection devices, monitoring system, DC system, remote back-end, and fault information processing system together, presenting the trainees a completely same working situation with the substation control room after the simulation server is started up.

The only difference with the real scene is the alternating current circuit. Considering the space and cost, all devices needing alternating current, including protection devices, monitoring and control unit, fault recording and bus differential protection are all connected into a roll. The connection makes thetraining on protection device standardization work different from the actual scene.

\section{Conclusion}

Substation relay protection device is one of the important partsin power system, providing critical security guarantee for the safe and stable operation of the power system. Professional operating skill level of substation workers is a key factor for the stable operation of relay protection devices and the safe and stable operation of the power system. The increasing number of substation relay protection devices and the constantly updating professional application technologies in power system also requires relay protection workers to improve their theoretical knowledge and skill s with the times. Therefore, it is required to provide substation workers with trainings on related theoretical knowledge and combining it with the operation in actual work.

\section{References}

[1] Chen Liyi, GuQiang. Digital Simulation and its Development in Power System[J]. Automation of Electric Power Systems, 1999, 23 (23): 1-6

[2] Huang Jiayu, Chen Liyi, Sun Dechang. Digital Simulation of Power System[J]. Beijing: China Water Power Press, 1995 
[3] ZhengSanli, Lei Xianzhang, D. Retzmann, Han Yingduo. Computer and Real-time Digital Simulation in Power System (first part) -Computer Simulationof Power System [M], Power System Automation, 2001, 25 (13): 45-49

[4] ZhengSanli, Lei Xianzhang, D. Retzmann, Han Yingduo. Computer and Real-time Digital Simulation in Power System (first part) -Computer Simulation of Power System [M], Power System Automation, 2001,25 (14): 40-44

[5] LiaoZiqiang, Huang Zhongxiang. Development of Integrated Automation Substation Simulation Training System [J].Hubei Electric Power. 2003, (4): 38-40 